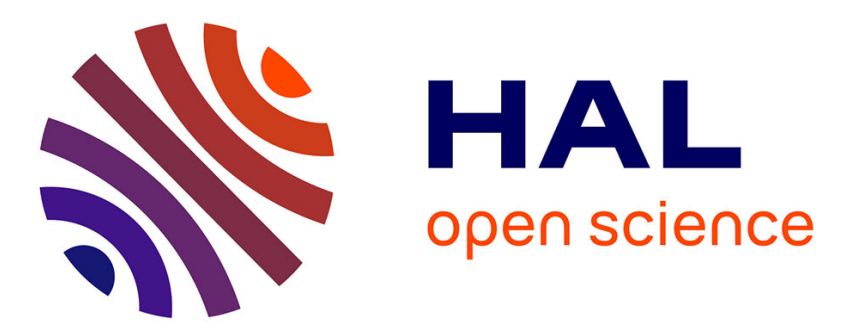

\title{
Graph theoretical properties of logic based argumentation frameworks: proofs and general results
}

Bruno Yun, Madalina Croitoru, Srdjan Vesic, Pierre Bisquert

\section{To cite this version:}

Bruno Yun, Madalina Croitoru, Srdjan Vesic, Pierre Bisquert. Graph theoretical properties of logic based argumentation frameworks: proofs and general results. 5th International Workshop on Graph Structures for Knowledge Representation and Reasoning (GKR 2017), Aug 2017, Melbourne, Australia. pp.118-138, 10.1007/978-3-319-78102-0_7 . lirmm-01892703

\section{HAL Id: lirmm-01892703 \\ https://hal-lirmm.ccsd.cnrs.fr/lirmm-01892703}

Submitted on 10 Oct 2018

HAL is a multi-disciplinary open access archive for the deposit and dissemination of scientific research documents, whether they are published or not. The documents may come from teaching and research institutions in France or abroad, or from public or private research centers.
L'archive ouverte pluridisciplinaire HAL, est destinée au dépôt et à la diffusion de documents scientifiques de niveau recherche, publiés ou non, émanant des établissements d'enseignement et de recherche français ou étrangers, des laboratoires publics ou privés. 


\title{
Graph theoretical properties of logic based argumentation frameworks: proofs and general results
}

\author{
Bruno Yun ${ }^{1}$, Madalina Croitoru ${ }^{1}$, Srdjan Vesic $^{2}$, Pierre Bisquert $^{3}$ \\ ${ }^{1}$ LIRMM, University of Montpellier, France \\ ${ }^{2}$ CRIL, University of Artois, France \\ ${ }^{3}$ INRA, France
}

\begin{abstract}
In this paper we extend our first results concerning the characterisation of the graph structure of logic based argumentation graphs with two main classes of findings. First we provide full proofs for the structural results of argumentation graphs built over Datalog+/- knowledge base composed of facts and negative constraints solely. Second, we also provide some structural properties for the general case of knowledge bases composed of facts, rules and negative constraints.
\end{abstract}

\section{Introduction}

We consider existential rules [Calì et al., 2009] as the underlying logical language for the argumentation framework. Starting from an inconsistent existential rule knowledge base (composed of a set of factual knowledge and an ontology stating positive and negative rules about the factual knowledge), using the instantiation of Croitoru and Vesic [2013] we generate the arguments and the attacks corresponding to the knowledge base. The instantiation has been proven to respect rationality desiderata [Amgoud, 2014: Caminada and Amgoud, 2007| from the argumentation literature and outputs a set of extensions equivalent to the repairs [Lembo et al. 2010, Bienvenu, 2012] of the knowledge base (i.e. the maximum w.r.t. inclusion consistent set of factual knowledge).

In Yun et al. [2018] we have given on overview of structural results for simple knowledge bases composed over facts and negative rules only (without any positive rules). In this paper we extend this first study by detailing the results on simple knowledge bases and providing some results that also hold in the general case. More precisely:

- We first consider the case of the knowledge base solely consisting of factual knowledge and negative constraints (expressing fact incompatibility). We fully prove the following structural properties of the argumentation graphs constructed from such knowledge bases: the existence of several duplicates of the same sub-graph, graphautomorphism induced graph symmetries and specific strongly connected component behaviour. We demonstrate how this serves as a complete characterisation of argumentation frameworks obtained from such knowledge bases. We also show that the cf2 semantics [Baroni et al., 2005, Gaggl and Woltran, 2013] coincides with the preferred and naive semantics in the case of argumentation frameworks generated 
from such knowledge bases (without positive rules) that only contain binary negative constraints. Furthermore, we give an example showing that if ternary negative constraints are added, this equivalence no longer holds.

- Second, for the general case of knowledge bases with any number of facts, rules or negative constraints we unveil the following structural properties: the presence of a complete directed sub-graph, the presence of at least one cycle in the graph and, intriguingly, the fact that the cf2 semantics [Baroni et al., 2005, Gaggl and Woltran, 2013] is yielding, in this instantiation, potentially inconsistent bases of arguments.

The significance of our results lies in the graph theoretical structural analysis of a whole family of potentially real world argumentation graphs. Such results are important to know for software engineers when designing argumentation solvers. For example, when designing SAT inspired solvers [Cerutti et al., 2013], graph symmetries induce as choice the solvers that better perform in the presence of symmetries [Lagniez et al. 2015]. Another practical interest lies in benchmark generation [Yun et al. 2017a]. Recently argumentation competitions [Thimm et al. [2016] have been held where the benchmarks are generated based on graph theoretical structures known to be difficult for solvers but not confirmed to appear in practice. Revealing real world behaviour could fill this gap and complete the benchmark spectra of instances. Last, it is important to be aware of logic based argumentation graph behaviour in order to keep a realistic expectation of the added value of argumentation in such domains. It is known [Yun et al., 2017b] that even for a modest knowledge base of 7 facts, 2 rules and 1 binary negative constraints, the generated argumentation graph can take gargantuan proportions reaching 383 arguments and 32768 attacks. This paper will help explain, at least partially, these results. For example, the sub-graph duplicate result directly shows the exponential growth of the argumentation graph when facts are added to the knowledge base. Please note that even if the paper of Yun et al. [2017b] deals with benchmark generation of existential rule knowledge bases it is fundamentally different from this work in at least two ways. First, no graph theoretical properties are demonstrated in Yun $e t$ al. [2017b]. Second, regarding practical added value of Yun et al. [2017b], the authors provide a benchmark generation tool but given the size of the generated graphs it would be difficult to generate the graphs in order to test for their structure. This paper fills this gap and directly provides a variety of graph theoretical properties such graphs enjoy.

In Section 2, we recall the basic notions of existential rules and argumentation. In Section 3 , we show a complete set of structural properties for argumentation frameworks generated from knowledge bases without rules w.r.t. symmetry, strongly connected components and $k$-copy graphs. Then, the rest of the section deals with structural results for argumentation frameworks generated from general knowledge bases. We show general structural properties such as the absence of self-attacking arguments but also the presence of complete directed sub-graphs. We conclude with a concrete example showing that the cf 2 semantics is not suitable for existentially rule instantiated logical argumentation frameworks as it can output sets with inconsistent bases. 


\section{Background Notions}

The existential rules language [Calì et al. 2009] has attracted much interest recently in the Semantic Web and Knowledge Representation community for its suitability for representing knowledge in a distributed context (such as Ontology Based Data Access (OBDA) applications) [Thomazo and Rudolph, 2014, Zhang et al., 2016]. It is composed of formulae built with the usual quantifiers $(\exists, \forall)$ and only two connectors: implication $(\rightarrow)$ and conjunction $(\wedge)$ and is composed of the following elements:

- A fact is a ground atom of the form $p\left(t_{1}, \ldots, t_{k}\right)$ where $p$ is a predicate of arity $k$ and $t_{i}, i \in[1, \ldots, k]$ constants.

- An existential rule is of the form $\forall \vec{X}, \vec{Y} H[\vec{X}, \vec{Y}] \rightarrow \exists \vec{Z} C[\vec{Z}, \vec{X}]$ where $H$ (called the hypothesis) and $C$ (called the conclusion) are existentially closed atoms or conjunctions of existentially closed atoms and $\vec{X}, \vec{Y}, \vec{Z}$ their respective vectors of variables. A rule is applicable on a set of facts $\mathcal{F}$ iff there exists a homomorphism from the hypothesis of the rule to $\mathcal{F}$. Applying a rule to a set of facts (also called chase) consists of adding the set of atoms of the conclusion of the rule to the facts according to the application homomorphism. Different chase mechanisms use different simplifications that prevent infinite redundancies [Baget et al. 2011]. We use recognisable classes of existential rules where the chase is guaranteed to stop [Baget et al. 2011].

- A negative constraint is a particular kind of rule where $C$ is $\perp$ (absurdum). Negative constraints can be of any arity (i.e. the number of atoms in $C$ is not bounded). Negative constraints implement weak negation. Please note that negative constraints generalise simple binary conflicts that can easily be translated between the two representations: $\neg p(\boldsymbol{X})$ is transformed into $n p(\boldsymbol{X})$ and the negative constraint $p(\boldsymbol{X}) \wedge n p(\boldsymbol{X}) \rightarrow \perp$ is added to the rules set.

- A knowledge base $\mathcal{K}=(\mathcal{F}, \mathcal{R}, \mathcal{N})$ is composed of a finite set of facts $\mathcal{F}$, a set of rules $\mathcal{R}$ and a set of negative constraints $\mathcal{N}$. We denote by $\mathcal{C} \ell_{\mathcal{R}}^{*}(\mathcal{F})$ the closure of $\mathcal{F}$ by $\mathcal{R}$ (computed by all possible rule $\mathcal{R}$ applications over $\mathcal{F}$ until a fixed point). $\mathcal{C} \ell_{\mathcal{R}}^{*}(\mathcal{F})$ is said to be $\mathcal{R}$-consistent if no negative constraint hypothesis can be deduced. Otherwise, $\mathcal{C} \ell_{\mathcal{R}}^{*}(\mathcal{F})$ is $\mathcal{R}$-inconsistent.

- Given a knowledge base $\mathcal{K}=(\mathcal{F}, \mathcal{R}, \mathcal{N})$, a set of facts $C \subseteq \mathcal{F}$ is called a minimal conflict iff $C$ is $\mathcal{R}$-inconsistent and any strict subset $C^{\prime} \subset C$ of it is $\mathcal{R}$-consistent. The set of all minimal conflict of $\mathcal{K}$ is denoted $\operatorname{conflicts}(\mathcal{K})$. If there are no minimal conflicts there are no attacks.

In the OBDA setting rules and constraints act as an ontology used to "access" different data sources. These sources are prone to inconsistencies. As per literature principles, we suppose that the set of rules is compatible with the set of negative constraints, i.e. the union of those two sets is satisfiable [Lembo et al. 2010]. This assumption is made because in OBDA we assume that the ontology is believed to be reliable as it is the result of a robust construction by domain experts. However, as data can be large and heterogeneous due to merging and fusion, in the OBDA setting the data is assumed to be the source of inconsistency. This means that by applying the rules on the set of facts, we might violate a constraint. To handle inconsistency, in this paper we use the existential rule instantiation of argumentation frameworks of Croitoru and Vesic [2013]: 
- An argument [Croitoru and Vesic, 2013] in Datalog ${ }^{ \pm}$is composed of a minimal (w.r.t. set inclusion) set of facts called support and a conclusion entailed from the support. The Skolem chase coupled with the use of decidable classes of Datalog ${ }^{ \pm}$ ensures the finiteness of the argumentation framework proposed (following from Baget et al. [2011]). Formally, an argument $a$ is a tuple $(H, C)$ with $H$ a non-empty $\mathcal{R}$-consistent subset of $\mathcal{F}$ and $C$ a set of facts:

- $H \subseteq \mathcal{F}$ and $\mathcal{C} \ell_{\mathcal{R}}^{*}(H) \not \models \perp$ (consistency)

- $C \subseteq \mathcal{C} \ell_{\mathcal{R}}^{*}(H)$ (entailment)

- $\nexists H^{\prime} \subset H$ s.t. $C \subseteq \mathcal{C} \ell_{\mathcal{R}}^{*}\left(H^{\prime}\right)$ (minimality)

The support $H$ of an argument $a$ is denoted by $\operatorname{Supp}(a)$ and the conclusion $C$ by $\operatorname{Conc}(a)$.

- The attack considered is the undermine [Croitoru and Vesic, 2013]: $a$ attacks $b$ iff the union of the conclusion of $a$ and an element of the support of $b$ are $\mathcal{R}$ inconsistent. Formally, an argument $a$ attacks an argument $b$ denoted by $(a, b) \in \mathcal{C}$ (or $a \mathcal{C} b$ ) iff $\exists \phi \in \operatorname{Supp}(b)$ s.t. $\operatorname{Conc}(a) \cup\{\phi\}$ is $\mathcal{R}$-inconsistent. The set of attackers of an argument $a$ is denoted $A_{t t}^{-}(a)=\left\{a^{\prime} \mid a^{\prime} \mathcal{C} a\right\}$ and the set of arguments attacked by $a, \operatorname{Att}^{+}(a)=\left\{a^{\prime} \mid a \mathcal{C} a^{\prime}\right\}$.

- An argumentation framework $\mathcal{A} \mathcal{S}_{\mathcal{K}}=(\mathcal{A}, \mathcal{C})$ is the corresponding AF of $\mathcal{K}$ where $\mathcal{A}$ is the set of arguments and $\mathcal{C}$ is the corresponding attack relation defined above.

- If $X$ is a set of arguments, $\operatorname{Base}(X)$ is the union of the supports of the arguments of $X: \operatorname{Base}(X)=\bigcup_{x \in X} \operatorname{Supp}(x)$.

Example 1. Let us consider the knowledge base $\mathcal{K}=(\mathcal{F}, \mathcal{R}, \mathcal{N})$ with:

$\mathcal{F}=\{a(m), b(m), c(m), d(m)\}, \mathcal{R}=\emptyset$ and $\mathcal{N}=\{\forall x(a(x) \wedge b(x) \wedge c(x) \rightarrow \perp)\}$.

The corresponding argumentation framework $\mathcal{A} \mathcal{S}_{\mathcal{K}}$ is composed of 36 attacks and the following 13 arguments:

- $a 0 \_0:(\{a(m)\},\{a(m)\})$

- $a 1_{-} 0:(\{b(m)\},\{b(m)\})$

- $a 2 \_2:(\{a(m), b(m)\},\{a(m), b(m)\})$

- a3_0: $(\{c(m)\},\{c(m)\})$

- $a 4 \_2:(\{a(m), c(m)\},\{a(m), c(m)\})$

- $a 5 \_2:(\{b(m), c(m)\},\{b(m), c(m)\})$

- a6_0 : $(\{d(m)\},\{d(m)\})$

- $a 7 \_2:(\{a(m), d(m)\},\{a(m), d(m)\})$

- $a 8 \_2:(\{b(m), d(m)\},\{b(m), d(m)\})$

- a9_6: $(\{a(m), b(m), d(m)\},\{a(m), b(m), d(m)\})$

- a10_2: $(\{c(m), d(m)\},\{c(m), d(m)\})$

- a11_6: $(\{a(m), c(m), d(m)\},\{a(m), c(m), d(m)\})$

- a12_6: $(\{b(m), c(m), d(m)\},\{b(m), c(m), d(m)\})$

Please note that the attack is not symmetric, for instance, the argument $a 5 \_2$ attacks the argument $a 0 \_0$ but not conversely.

Let us now recall basic argumentation notions [Dung, 1995]. Let $\mathcal{A S}$ be an argumentation framework, $\mathrm{S} \subseteq \mathcal{A}$ and $a \in \mathcal{A}$. We say that:

- $\mathrm{S}$ is conflict-free iff there exists no arguments $a, b \in \mathrm{S}$ such that $(a, b) \in \mathcal{C}$. 
- S defends $a$ iff for every argument $b \in \mathcal{A}$, if we have $(b, a) \in \mathcal{C}$ then there exists $c \in \mathrm{S}$ such that $(c, b) \in \mathcal{C}$.

- $\mathrm{S}$ is admissible iff it is conflict-free and defends all its arguments.

- $\mathrm{S}$ is a preferred extension iff it is a maximal (with respect to set inclusion) admissible set.

- $\mathrm{S}$ is a stable extension iff it is conflict-free and for all $a \in \mathcal{A} \backslash \mathrm{S}$, there exists an argument $b \in \mathrm{S}$ such that $(b, a) \in \mathcal{C}$.

Example 2 (cont.). There are 3 stable (resp. preferred) extensions in $\mathcal{A S}_{\mathcal{K}}$ :

- $\varepsilon_{1}=\left\{a 0 \_0, a 1 \_0, a 2 \_2, a 6 \_0, a 7 \_2, a 8 \_2, a 9 \_6\right\}$

- $\varepsilon_{2}=\left\{a 1 \_0, a 3 \_0, a 5 \_2, a 6 \_0, a 8 \_2, a 10 \_2, a 12 \_6\right\}$

- $\varepsilon_{3}=\left\{a 0 \_0, a 3 \_0, a 4 \_2, a 6 \_0, a 7 \_2, a 10 \_2, a 11 \_6\right\}$

It was shown in Croitoru and Vesic [2013] that, for existential rules argumentation frameworks, the set of preferred and stable extensions coincide and correspond to the set of maximally consistent sets of facts (repairs).

Example 3 (cont.). The preferred extension $\varepsilon_{1}$ corresponds to the repair: $r_{1}=\{a(m), b(m), d(m)\}$.

Indeed, we have that $\operatorname{Base}\left(\varepsilon_{1}\right)=r_{1}$.

\section{Structural Results}

This section is organised as follows. In Section 3.1 we first investigate the graph theoretical results of knowledge bases composed solely of facts and negative constraints. Then, in Section 3.2 we investigate the general case where rules are also considered in the argumentation framework.

\subsection{Results for simple knowledge bases}

The graph theoretical results of this subsection are solely looking at the case where the knowledge base is composed of a set of facts and a set of negative constraints defined on these facts. Therefore, at the basis of the results lies the notion of knowledge base minimal conflict. We exhibit three main results:

- The first result deals with conflict induced structural properties. Namely, we characterise dummy arguments, arguments that are un-attacked and that do not attack other arguments, and show the repetitious nature of the argumentation graph by introducing the notion of $k$-copy graph.

- The second result deepens these results and looks into the symmetries of the argumentation graph based on graph auto-morphisms.

- Last, we look into the connectivity of the graph and demonstrate strongly connected components related results.

Please note that these three points will enable us to completely characterise the structural properties of argumentation graphs generated from knowledge bases without positive rules. We begin by introducing the scope of a negative constraint which is the set of all sets of facts on which the negative constraint is applicable. 
Definition 1. Let $\mathcal{K}=(\mathcal{F}, \mathcal{R}, \mathcal{N})$ be a knowledge base with $\mathcal{R}=\emptyset$ and $N \in \mathcal{N}$ be a negative constraint. We define the scope of the negative constraint $N$ as the set $\mathcal{F}_{N}=\{X \subseteq \mathcal{F} \mid X$ is minimal with respect to set inclusion such that there is an homomorphism from the body of $N$ to $X\}$.

Example 4 (cont.). The scope of the negative constraint $N=\forall x(a(x) \wedge b(x) \wedge c(x) \rightarrow$ $\perp$ is $\mathcal{F}_{N}=\{\{a(m), b(m), c(m)\}\}$.

We show that the number of un-attacked arguments that do not attack other arguments, called "dummy arguments", depends on the number of facts and the scope of all negative constraints.

Proposition 1. Let $\mathcal{K}=(\mathcal{F}, \mathcal{R}, \mathcal{N})$ be an inconsistent knowledge base such that $\mathcal{R}=\emptyset$ and $|\mathcal{F}|=n$. If $\mathcal{A} \mathcal{S}_{\mathcal{K}}=(\mathcal{A}, \mathcal{C})$ is the corresponding argumentation framework, there are exactly $2^{n-k}-1$ dummy arguments a in $\mathcal{A} \mathcal{S}_{\mathcal{K}}$ such $k=\left|\bigcup_{N \in \mathcal{N}} \bigcup_{X \in \mathcal{F}_{N}} X\right|$.

Proof. Denote $J=\bigcup_{N \in \mathcal{N}} \bigcup_{X \in \mathcal{F}_{N}} X$.

Denote $U n n=\left\{a \in \mathcal{A} \mid \operatorname{Att}^{-}(a)=\operatorname{Att}^{+}(a)=\emptyset\right\}$.

1. Let us prove that $|U n n| \geq 2^{n-k}-1$ with $|J|=k$ and $|\mathcal{F}|=n$. The set $J=$ $\bigcup_{N \in \mathcal{N}} \bigcup_{X \in \mathcal{F}_{N}} X$ corresponds to the set of facts that trigger at least one negative constraint. Thus, every fact that belongs to $E=\mathcal{F} \backslash J$ is not in any conflict. Since $|E|=n-k$ and $\mathcal{R}=\emptyset$, we conclude that there are at least $2^{n-k}-1$ arguments that have a non empty subset of $E$ as support. These arguments are not attacked and do not attack other arguments as the elements of their supports and conclusions are not in any conflict.

2. Let us prove that $|U n n| \leq 2^{n-k}-1$ with $|J|=k$ and $|\mathcal{F}|=n$. By means of contradiction, suppose that there is an argument $a$ that do not attack other arguments and that is not attacked but $\operatorname{Supp}(a) \nsubseteq E$. It means there exists a negative constraint $N$ such that $\left(\bigcup_{X \in \mathcal{F}_{N}} X\right) \cap \operatorname{Supp}(a) \neq \emptyset$ and there is $X \in \mathcal{F}_{N}$ such that $X \cap \operatorname{Supp}(a) \neq \emptyset$. Now, let us consider $Y=X \backslash \operatorname{Supp}(a)$. We know that $Y$ is not empty otherwise there is a contradiction with the consistency of the support of $a$. Furthermore, $Y$ is $\mathcal{R}$-consistent since $|Y|$ is strictly inferior to the arity of the negative constraint $N$. Thus, there is an argument $b=(Y, Y)$ such that $(b, a) \in \mathcal{C}$, contradiction. 
Example 5 (cont.). We have that $n=4$ and since we know that $\bigcup_{N \in \mathcal{N}} \bigcup_{X \in \mathcal{F}_{N}} X=$ $\{a(m), b(m), c(m)\}$, we conclude that there is $2^{4-3}-1=1$ dummy argument. This argument corresponds to $a 6_{-} 0=(\{d(m)\},\{d(m)\})$.

We now analyse the related behaviour of atoms in the scope of a negative constraint. To do so we introduce the notion of $k$-copy graph.

A $k$-copy graph of a graph is another graph that has $k$ times more arguments and each copy $a^{\prime}$ of $a$ attacks the same arguments as $a$ and possesses the same attackers. Formally:

Definition 2. Let $\mathcal{A S}=(\mathcal{A}, \mathcal{C})$ be an argumentation framework. We say that the graph of $\mathcal{A S}$ is a k-copy graph of $\mathcal{A} \mathcal{S}^{\prime}=\left(\mathcal{A}^{\prime}, \mathcal{C}^{\prime}\right)$ iff:

- $|\mathcal{A}|=k *\left|\mathcal{A}^{\prime}\right|$ and there is a surjective function $f$ from $\mathcal{A}$ to $\mathcal{A}^{\prime}$ such that for every argument $a^{\prime} \in \mathcal{A}^{\prime}$, we have $\left|W_{a^{\prime}}\right|=k$, where $W_{a^{\prime}}=\left\{a \in \mathcal{A} \mid f(a)=a^{\prime}\right\}$.

- For all $a, b \in \mathcal{A},(a, b) \in \mathcal{C}$ iff $(f(a), f(b)) \in \mathcal{C}^{\prime}$.

Example 6. In Figure 1 the graph $G^{\prime}$ (on the right) is a 2-copy graph of the graph $G$ (on the left). We have that $W_{a}=\left\{a_{1}^{\prime}, a_{2}^{\prime}\right\}, W_{b}=\left\{b_{1}^{\prime}, b_{2}^{\prime}\right\}, W_{c}=\left\{c_{1}^{\prime}, c_{2}^{\prime}\right\}$.
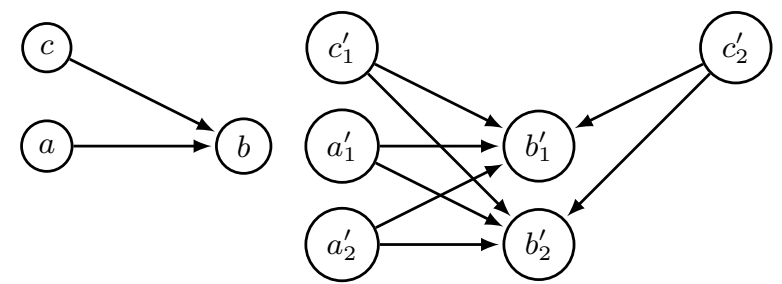

Fig. 1: Representation of a 2-copy graph.

If two arguments are the copies of the same argument, then they attack the same arguments and are attacked by the same arguments.

The following proposition shows that if there is a knowledge base $\mathcal{K}$ with no rule and containing $k$ facts that are not in the scope of any negative constraints, then there exists a subgraph of $\mathcal{A S} \mathcal{S}_{\mathcal{K}}$ that is a $2^{k}$-copy graph of $\mathcal{A} \mathcal{S}_{\mathcal{K}^{\prime}}$ where $\mathcal{K}^{\prime}$ is the knowledge base with no rules, the same negative constraints as $\mathcal{K}$ and that contains only the facts that are in the scope of at least one negative constraint of $\mathcal{K}$.

This property is important as it shows the behaviour of the instantiation in the case of addition of facts not appearing in any conflict. It shows the structure of the graph and exhibits the exponential growth of the number of arguments w.r.t. these facts. 
Proposition 2. Let $\mathcal{K}=(\mathcal{F}, \mathcal{R}, \mathcal{N})$ be a knowledge base with $\mathcal{R}=\emptyset$.

If $J=\bigcup_{N \in \mathcal{N}} \bigcup_{X \in \mathcal{F}_{N}} X \neq \emptyset$ and $|\mathcal{F} \backslash J|=k$ then there is a subgraph of $\mathcal{A} \mathcal{S}_{\mathcal{K}}=$ $(\mathcal{A}, \mathcal{C})$ that is a $\left(2^{k}\right)$-copy graph of $\mathcal{A S}_{\mathcal{K}^{\prime}}=\left(\mathcal{A}^{\prime}, \mathcal{C}^{\prime}\right)$ where $\mathcal{K}^{\prime}=(J, \mathcal{R}, \mathcal{N})$ and $|\mathcal{A}|=\left(\left|\mathcal{A}^{\prime}\right|+1\right) * 2^{k}-1$.

Proof. If $|\mathcal{F} \backslash J|=0$, then it is obvious that $\mathcal{A S} \mathcal{S}_{\mathcal{K}}$ is a 1-copy graph of itself. Suppose now that $|\mathcal{F} \backslash J|>0$. We denote by $\mathcal{A} \mathcal{S}_{\mathcal{K}^{\prime}}=\left(\mathcal{A}^{\prime}, \mathcal{C}^{\prime}\right)$ the argumentation framework from the knowledge base $\mathcal{K}^{\prime}=(J, \mathcal{R}, \mathcal{N})$. Moreover, since $\mathcal{R}=\emptyset$, the arguments can only be of the form $(X, X)$ where $X$ is an $\mathcal{R}$-consistent subset of $J$. Hence, $\left|\mathcal{A}^{\prime}\right|=$ $\mid\{X \mid X$ is an $\mathcal{R}$-consistent subset of $J\} \mid$.

Now, let us consider $\mathcal{A S} \mathcal{S}_{\mathcal{K}}=(\mathcal{A}, \mathcal{C})$, the argumentation framework corresponding to the knowledge base $\mathcal{K}=(\mathcal{F}, \mathcal{R}, \mathcal{N})$. We show that the subgraph $\mathcal{A} \mathcal{S}_{\mathcal{K}}^{\prime \prime}=\left(\mathcal{A}^{\prime \prime}, \mathcal{C}^{\prime \prime}\right)$ of $\mathcal{A} \mathcal{S}_{\mathcal{K}}$ where $\mathcal{A}^{\prime \prime}=\{a \in \mathcal{A} \mid \operatorname{Supp}(a) \cap J \neq \emptyset\}$ and $\mathcal{C}^{\prime \prime}=\mathcal{C}_{\mid \mathcal{A}^{\prime \prime}}$ is a $\left(2^{|\mathcal{F} \backslash J|}\right)$-copy graph of $\mathcal{A} \mathcal{S}_{\mathcal{K}^{\prime}}$ :

- We know that for any set $X$ that is an $\mathcal{R}$-consistent subset of $J, X \cup X^{\prime}$, where $X^{\prime}$ is a subset of $\mathcal{F} \backslash J$, is an $\mathcal{R}$-consistent set. Thus $\left|\mathcal{A}^{\prime \prime}\right|=\mid\left\{X \cup X^{\prime} \mid X^{\prime} \subseteq \mathcal{F} \backslash J\right.$ and $X$ is an $\mathcal{R}$-consistent subset of $J\} \mid$. Since the number of subsets of $\mathcal{F} \backslash J$ is $2^{|\mathcal{F} \backslash J|}$, then $\left|\mathcal{A}^{\prime \prime}\right|=\left|\mathcal{A}^{\prime}\right| * 2^{|\mathcal{F} \backslash J|}$.

- We denote by $f$ the function from $\mathcal{A}^{\prime \prime}$ to $\mathcal{A}^{\prime}$ such that $f\left(a^{\prime \prime}\right)=a^{\prime}$ iff $\operatorname{Supp}\left(a^{\prime}\right)=$ $\operatorname{Supp}\left(a^{\prime \prime}\right) \cap J$. We now show that this function is surjective. Let $a^{\prime}$ be an argument of $\mathcal{A}^{\prime}$ and $c$ an arbitrary element of $\mathcal{F} \backslash J$ (it exists since $|\mathcal{F} \backslash J|>0$ ). As mentioned before, we know that $E=\operatorname{Supp}\left(a^{\prime}\right) \cup\{c\}$ is $\mathcal{R}$-consistent. Therefore $a^{\prime \prime}=(E, E)$ is an argument of $\mathcal{A}^{\prime \prime}$ and $f\left(a^{\prime \prime}\right)=a^{\prime}$.

- Let $a^{\prime} \in \mathcal{A}^{\prime}$ and $W_{a^{\prime}}=\left\{a^{\prime \prime} \in \mathcal{A}^{\prime \prime} \mid f\left(a^{\prime \prime}\right)=a^{\prime}\right\}$. For every subset $X$ of $\mathcal{F} \backslash J$, $L=X \cup \operatorname{Supp}\left(a^{\prime}\right),(L, L) \in W_{a^{\prime}}$. Since the number of different subsets of $\mathcal{F} \backslash J$ is $2^{|\mathcal{F} \backslash J|}$, we have $\left|W_{a^{\prime}}\right| \geq 2^{|\mathcal{F} \backslash J|}$. Since for every $a_{1}^{\prime}, a_{2}^{\prime} \in A^{\prime}, W_{a_{1}^{\prime}} \cap W_{a_{2}^{\prime}}=\emptyset$, then for every $a^{\prime} \in \mathcal{A}^{\prime},\left|W_{a^{\prime}}\right|=2^{|\mathcal{F} \backslash J|}$ because $\left|\mathcal{A}^{\prime \prime}\right|=\left|\mathcal{A}^{\prime}\right| * 2^{|\mathcal{F} \backslash J|}$.

- Let $\left(a_{1}^{\prime \prime}, a_{2}^{\prime \prime}\right) \in \mathcal{C}^{\prime \prime}$, by definition, we have that there exists $\phi \in \operatorname{Supp}\left(a_{2}^{\prime \prime}\right)$ s.t. $\operatorname{Conc}\left(a_{1}^{\prime \prime}\right) \cup\{\phi\}$ is $\mathcal{R}$-inconsistent. Since there are no rules, it is true that $\operatorname{Supp}\left(a_{1}^{\prime \prime}\right) \cup$ $\{\phi\}$ is also $\mathcal{R}$-inconsistent. However, it is clear that this inconsistency cannot come from elements of $\mathcal{F} \backslash J$. Thus, there exists $\phi \in \operatorname{Supp}\left(a_{2}^{\prime \prime}\right) \cap J$ such that $\left(\operatorname{Supp}\left(a_{1}^{\prime \prime}\right) \cap\right.$ $J) \cup\{\phi\}$ is $\mathcal{R}$-inconsistent. Therefore $\left(f\left(a_{1}^{\prime \prime}\right), f\left(a_{2}^{\prime \prime}\right)\right) \in \mathcal{C}^{\prime}$ since $\operatorname{Supp}\left(f\left(a_{1}^{\prime \prime}\right)\right)=$ $\operatorname{Supp}\left(a_{1}^{\prime \prime}\right) \cap J$ and $\operatorname{Supp}\left(f\left(a_{2}^{\prime \prime}\right)\right)=\operatorname{Supp}\left(a_{2}^{\prime \prime}\right) \cap J$.

- Let $a_{1}^{\prime \prime}, a_{2}^{\prime \prime} \in \mathcal{A}^{\prime \prime}$ such that $\left(f\left(a_{1}^{\prime \prime}\right), f\left(a_{2}^{\prime \prime}\right)\right) \in \mathcal{C}^{\prime}$. It means that there exists $\phi \in$ $\operatorname{Supp}\left(f\left(a_{2}^{\prime \prime}\right)\right)$ s.t. $\operatorname{Conc}\left(f\left(a_{1}^{\prime \prime}\right)\right) \cup\{\phi\}$ is $\mathcal{R}$-inconsistent. By definition, we have that $\operatorname{Supp}\left(f\left(a_{2}^{\prime \prime}\right)\right)=\operatorname{Supp}\left(a_{2}^{\prime \prime}\right) \cap J$, thus $\phi \in \operatorname{Supp}\left(a_{2}^{\prime \prime}\right)$. Likewise, we have that $\operatorname{Conc}\left(f\left(a_{1}^{\prime \prime}\right)\right)=\operatorname{Supp}\left(f\left(a_{1}^{\prime \prime}\right)\right)=\operatorname{Supp}\left(a_{1}^{\prime \prime}\right) \cap J=\operatorname{Conc}\left(a_{1}^{\prime \prime}\right) \cap J$. We conclude that $\left(\operatorname{Conc}\left(a_{1}^{\prime \prime}\right) \cap J\right) \cup\{\phi\}$ is $\mathcal{R}$-inconsistent. Therefore $\operatorname{Conc}\left(a_{1}^{\prime \prime}\right) \cup\{\phi\}$ is $\mathcal{R}$ inconsistent and $\left(a_{1}^{\prime \prime}, a_{2}^{\prime \prime}\right) \in \mathcal{C}^{\prime \prime}$.

Finally, we have that $|\mathcal{A}|=\mid\{X \mid X$ is an $\mathcal{R}$-consistent subset of $\mathcal{F}\}|=|\{X \mid$ $X \cap J \neq \emptyset$ and $X$ is an $\mathcal{R}$-consistent subset of $\mathcal{F}\} \cup\{X \mid X \subseteq \mathcal{F} \backslash J$ and $X$ is an $\mathcal{R}$-consistent subset of $\mathcal{F}\}|-1=| \mathcal{A}^{\prime} \mid * 2^{|\mathcal{F} \backslash J|}+2^{|\mathcal{F} \backslash J|}-1=\left(\left|\mathcal{A}^{\prime}\right|+1\right) * 2^{|\mathcal{F} \backslash J|}-1$. This concludes the proof. 
We want to emphasise the result of Proposition 2 as it shows that the addition of "superfluous" facts will increase the size of the argumentation graph by an exponential factor.

Example 7 (cont.). The argumentation framework $\mathcal{A} \mathcal{S}_{\mathcal{K}}$ has a subgraph that is a 2-copy graph of $\mathcal{A S}_{\mathcal{K}^{\prime}}$, where $\mathcal{K}^{\prime}=(\{a(m), b(m), c(m)\}, \emptyset, \mathcal{N})$. Indeed, the argumentation framework $\mathcal{A S}_{\mathcal{K}^{\prime}}$ is composed of the following arguments:

$$
\begin{aligned}
& \text { - } a_{1}:(\{a(m)\},\{a(m)\}) \\
& \text { - } a_{2}:(\{b(m)\},\{b(m)\}) \\
& \text { - } a_{3}:(\{a(m), b(m)\},\{a(m), b(m)\}) \\
& \text { - } a_{4}:(\{c(m)\},\{c(m)\}) \\
& \text { - } a_{5}:(\{a(m), c(m)\},\{a(m), c(m)\}) \\
& \text { - } a_{6}:(\{b(m), c(m)\},\{b(m), c(m)\})
\end{aligned}
$$

We have that $W_{a_{1}}=\left\{a 0 \_0, a 7 \_2\right\}$.

We now focus on detecting symmetries in the graph. Please first note that we have the presence of symmetric arcs in the argumentation framework without rules. It obviously holds that if all negative constraints are binary, then the graph has only symmetric arcs (since the undermining will rely on binary sets). However, if the set of rules is not empty the symmetry no longer holds.

We now explore the link between the instantiation and symmetries in graphs. The next definitions introduce the notions needed to comprehend symmetries, namely, permutations of arguments, orbit of an argument and the cycle notation of a permutation.

Definition 3. A permutation on a set of elements $X$ is a bijection $\sigma$ from $X$ to $X$. Given a permutation $\sigma$, the orbit of element $x \in X$ is the set $\mathcal{O}_{x}=\left\{x, \sigma(x), \sigma^{2}(x), \ldots, \sigma^{n}(x)\right\}$, with $n \in\{0,1, \ldots\}$ the minimal integer s.t. $\sigma^{n+1}(x)=x$.

Definition 4. Given a permutation $\sigma$ on $X$, an orbit $\mathcal{O}$ and an element $x \in \mathcal{O}$, a cycle is a sequence $\left(x, \sigma(x), \sigma^{2}(x) \ldots, \sigma^{n}(x)\right)$, where $n \in\{0,1, \ldots\}$ is the minimal integer such that $\sigma^{n+1}(x)=x$.

A permutation can be compactly expressed as a product of cycles corresponding to the orbits of the permutation 1

Definition 5. Let $G=(V, E)$ be a graph. A permutation $\sigma$ on set $V$ is an automorphism of $G$ iff for every two nodes $v_{1}, v_{2} \in V$, we have that $\left(v_{1}, v_{2}\right) \in E$ iff $\left(\sigma\left(v_{1}\right), \sigma\left(v_{2}\right)\right) \in E$.

The set of automorphisms of a graph, together with the function composition operator, form a group called the automorphism group. The automorphism groups of a graph characterise its symmetries, and are therefore very useful in determining certain of its properties. A subset of a group is called a generating set of a group iff every group's element can be expressed as the combination (under group operation) of finitely many elements of the subset and their inverses.

\footnotetext{
${ }^{1}$ In the rest of the paper, and in order to simplify the notation, we omit cycles corresponding to singleton orbits.
} 
Proposition 3. Let $\mathcal{A S}=(\mathcal{A}, \mathcal{C})$ be a k-copy graph of $\mathcal{A S}^{\prime}=\left(\mathcal{A}^{\prime}, \mathcal{C}^{\prime}\right)$. For every $a^{\prime} \in \mathcal{A}^{\prime}$, for every $a_{1}, a_{2}$ in $W_{a^{\prime}}$, we have that $\left(a_{1}, a_{2}\right)$ is an automorphism of $\mathcal{A S}$.

The next proposition shows that if we add nodes (and no arc) to a graph with automorphisms, then the obtained graph also has automorphisms. It is used for showing, in Proposition 5, that a graph constructed on a KB with no rules possesses non trivial automorphisms derived from its subgraph.

Proposition 4. Let $G=(V, E)$ be a graph such that $\sigma$ is an automorphism of $G$. The graph $G^{\prime}=(V \cup X, E)$, where $X \cap V=\emptyset$, has the automorphism $\sigma^{\prime}$ from $V \cup X$ to $V \cup X$ :

$$
\forall v \in V \cup X, \sigma^{\prime}(v)=\left\{\begin{aligned}
\sigma(v) & \text { if } v \in V \\
v & \text { if } v \in X
\end{aligned}\right.
$$

Proposition 5. Let $\mathcal{K}=(\mathcal{F}, \mathcal{R}, \mathcal{N})$ with $\mathcal{R}=\emptyset, J=\bigcup_{N \in \mathcal{N}} \bigcup_{X \in \mathcal{F}_{N}} X \neq \emptyset, \mid \mathcal{F} \backslash$ $J \mid=k, \mathcal{K}^{\prime}=(J, \mathcal{R}, \mathcal{N})$ and $\mathcal{A S}^{\prime \prime}$ be a $\left(2^{k}\right)$-copy graph of $\mathcal{A S}_{\mathcal{K}^{\prime}}=\left(\mathcal{A}^{\prime}, \mathcal{C}^{\prime}\right)$. If $\mathcal{A S}^{\prime \prime}$ has $k^{\prime}$ automorphisms, then $\mathcal{A S}_{\mathcal{K}}$ has at least $k^{\prime}$ automorphisms.

Proof. From Proposition 2, we know that $\mathcal{A} \mathcal{S}_{\mathcal{K}}$ has a subgraph $\mathcal{A} \mathcal{S}_{\mathcal{K}}^{\prime \prime}=\left(\mathcal{A}^{\prime \prime}, \mathcal{C}^{\prime \prime}\right)$ that is a $2^{k}$-copy graph of $\mathcal{A S}_{\mathcal{K}^{\prime}}$. We first show that every argument $a$ that is in $\mathcal{A} \backslash \mathcal{A}^{\prime \prime}$ is such that $\operatorname{Att}^{-}(a)=A t t^{+}(a)=\emptyset$. Then we use Proposition 4

1. We showed in the proof of Proposition 2 that $\mathcal{A}^{\prime \prime}=\{a \in \mathcal{A} \mid \operatorname{Supp}(a) \cap J \neq \emptyset\}$. Thus, $\mathcal{A} \backslash \mathcal{A}^{\prime \prime}=\{a \in \mathcal{A} \mid \operatorname{Supp}(a) \subseteq \mathcal{F} \backslash J\}$. Since we have no rules, the arguments in $\mathcal{A} \backslash \mathcal{A}^{\prime \prime}$ cannot attack other arguments.

2. From Proposition 4, we conclude that there is an automorphism of $\mathcal{A S} \mathcal{S}_{\mathcal{K}}$ for every automorphism of $\mathcal{A} \mathcal{S}_{\mathcal{K}}^{\prime \prime}$.

Proposition 5 is important as it shows that the graph inherit all of the automorphisms of its subgraph. This will be useful when designing new solvers relying on symmetries.

Example 8 (cont.). Using Proposition 4 and Proposition 5 , we have that $\left(a 0 \_0, a 7 \_2\right)$ is an automorphism of $\mathcal{A} \mathcal{S}_{\mathcal{K}}$.

We now characterise the connectivity of the graph by showing the structure of the strongly connected components. We first define the impossible set associated to a minimal conflict $C$ as the set containing all the possible subsets of $\mathcal{F}$ that are supersets of at least one subset of $C$ of size $|C-1|$.

Definition 6. Let $K$ be a knowledge base and $C$ a minimal conflict of conflicts $(\mathcal{K})$. The impossible set of $C$ denoted by $\operatorname{Imp}(C)$ is $\left\{X \subseteq \mathcal{F} \mid X^{\prime} \subseteq X\right.$ and $X^{\prime} \subseteq C$ with $\left.\left|X^{\prime}\right|=|C-1|\right\}$.

An argumentation framework is strongly connected if and only if there is a path from any argument $a$ to any argument $a^{\prime}$.

Definition 7. Let $\mathcal{A S}=(\mathcal{A}, \mathcal{C})$ be an $A F$. We say that $\mathcal{A S}$ is strongly connected iff for every $a, a^{\prime} \in \mathcal{A}^{\prime}$ such that $a \neq a^{\prime}$, there is a path from argument a to argument $a^{\prime}$. 
Please note that the set of nodes of any arbitrary directed graph can be partitioned such that the subgraphs, induced by each set of nodes, is strongly connected that are called the strongly connected components of this graph. In the rest of this paper, we will denote by $S C C(\mathcal{A S})$, this particular partition of the set of arguments of $\mathcal{A S}$.

In the following proposition, we characterise the structure of the strongly connected components of an argumentation framework obtained from a knowledge base without rules.

Proposition 6. Let $K B$ be a knowledge base such that $\mathcal{R}=\emptyset$ and $\mathcal{A} \mathcal{S}_{\mathcal{K}}=(\mathcal{A}, \mathcal{C})$ the corresponding AF. We have that:

1. $\left\{\left(X_{i}, X_{i}\right)\right\} \in S C C\left(\mathcal{A S} \mathcal{S}_{\mathcal{K}}\right)$ where $X_{i} \in 2^{\mathcal{F}} \backslash \bigcup_{C \in \operatorname{conflicts}(\mathcal{K})} \operatorname{Imp}(C)$

2. $\left(\mathcal{A} \backslash \bigcup_{i} s_{i}\right) \in S C C\left(\mathcal{A S} \mathcal{K}_{\mathcal{K}}\right)$

Proof. We split the proof in two parts:

1. Suppose that $s_{i}$ is not a strongly connected component by itself, it means that there is another argument $a$ such that there is a path from $x_{i}=\left\{X_{i}, X_{i}\right\}$ to $a$ and inversely. Let us denote by $a_{1}$, the first argument attacked by $x_{i}$ on a path from $x_{i}$ to $a$. By definition, it means that there exists $\phi \in S u p p\left(a_{1}\right)$ such that $X_{i} \cup\{\phi\}$ is $\mathcal{R}$-inconsistent. Since $X_{i}$ is $\mathcal{R}$-consistent, it means that $X_{i} \cup\{\phi\}$ is a minimal conflict and that $X_{i} \in \operatorname{Imp}\left(X_{i} \cup\{\phi\}\right)$. Then, $X i \notin 2^{\mathcal{F}} \backslash \bigcup_{C \in \operatorname{conflicts}(\mathcal{K})} \operatorname{Imp}(C)$, contradiction.

2. Let $a, b$ be two arguments in $\left(\mathcal{A} \backslash \bigcup_{i} s_{i}\right)$, we show here that there is a path from $a$ to $b$. From the definitions, we know that $a(\operatorname{resp} . b)$ is of the form $(X, X)\left(\operatorname{resp} .\left(X^{\prime}, X^{\prime}\right)\right)$ such that there exists a minimal conflict $C$ (resp. $\left.C^{\prime}\right)$ and $W \subseteq C$ (resp. $W^{\prime} \subseteq C^{\prime}$ ) with $|W|=|C-1|$ (resp. $\left|W^{\prime}\right|=\left|C^{\prime}-1\right|$ ) and $W \subseteq X$ (resp. $W^{\prime} \subseteq X^{\prime}$ ).

Let $H=C \backslash X, X^{\prime \prime}=X^{\prime} \backslash H, W^{\prime \prime} \subseteq X^{\prime \prime}$ with $\left|W^{\prime \prime}\right|=\left|X^{\prime \prime}-1\right|$ and $J=$ $H \cup W^{\prime \prime} \cup\left(C^{\prime} \backslash X^{\prime}\right)$.

- If $J$ is $\mathcal{R}$-consistent, we denote by $u$, the argument $(J, J)$. We have that $u$ belongs to $\left(\mathcal{A} \backslash \bigcup_{i} s_{i}\right)$ because $J=\left|C^{\prime}-1\right|$ and $J \subseteq C^{\prime}$. We have that $a$ attacks $u$ and $u$ attacks $b$.

- If $J$ is $\mathcal{R}$-inconsistent, it means that there is a minimal conflict $C^{\prime \prime} \subseteq J$ such that $C^{\prime \prime} \nsubseteq C^{\prime}$ and $C^{\prime \prime} \nsubseteq C$. Let us consider $K, L \subseteq J$ such that $|K|=|L|=$ $|J-1|, H \subseteq K$ and $H \nsubseteq L$. By definition, $K$ and $L$ are $\mathcal{R}$-consistent, thus the arguments $c=(K, K)$ and $d=(L, L)$ exist. We have that $a$ attacks $c, c$ attacks $d$ and $d$ attacks $b$.

Corollary 1. Let $K B$ be a knowledge base such that $\mathcal{R}=\emptyset$.

There are $\left|2^{\mathcal{F}} \backslash \bigcup_{C \in \text { conflicts }(\mathcal{K})} \operatorname{Imp}(C)\right|+1$ strongly connected components in $\mathcal{A S} \mathcal{S}_{\mathcal{K}}$

Example 9 (cont.).

The only minimal conflicts is $C_{1}=\{a(m), b(m), c(m)\}$.

We conclude that $2^{\mathcal{F}} \backslash \bigcup_{C \in \text { conflicts }(\mathcal{K})} \operatorname{Imp}(C)=\{\{a(m)\},\{b(m)\},\{c(m)\}$, $\{d(m)\},\{a(m), d(m)\},\{b(m), d(m)\},\{c(m), d(m)\}\}$ and that there are $7+1=8$ strongly connected components in $\mathcal{A S}$ 的. 
We now summarise all the structural properties of the AFs generated from simple knowledge bases using Figure 2 as an example:

- There is one $k$-copy graph (encircled in the dashed-line zone).

- The arguments that are not inside the $k$-copy graph are "dummy arguments" (arguments that are outside the dashed-line zone) and their number can be computed using Proposition 1 .

- There is one dense strongly connected component composed of the majority of the arguments (encircled in the grey circle).

- The other strongly connected components are composed of only one argument each (arguments that are outside of the grey circle). The number of strongly connected components can be computed using Corollary 1

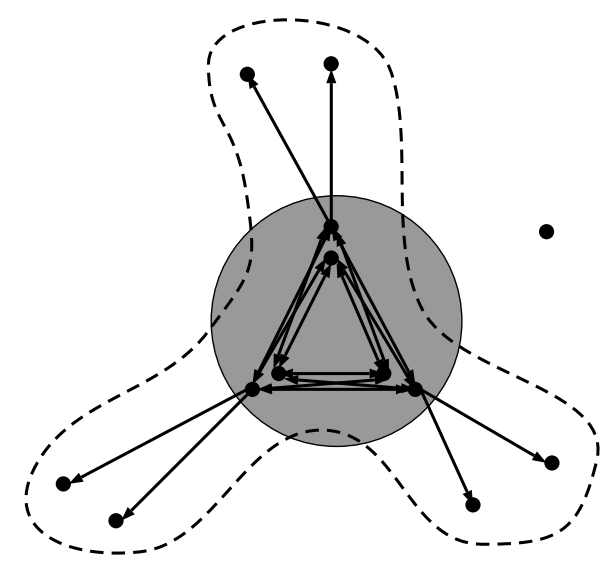

Fig. 2: Structural properties of AFs from simple KBs

Since we deal with strongly connected components, one of the research questions that naturally arise from this is whether or not the cf2 semantics [Baroni et al. 2011; Gaggl and Woltran, 2013] is equivalent to the preferred semantics in argumentation graphs generated from knowledge bases without positive rules.

On one hand, it appears that if the set of negative constraints is only composed of binary negative constraints, then the graph only has symmetric arcs. We conclude that since all SCCs are isolated, the cf 2 semantics coincides with the naive and preferred semantics.

Proposition 7. Let $K B$ be a knowledge base such that $\mathcal{R}=\emptyset$, then the cf 2 semantics coincides with the preferred and the naive semantics in $\mathcal{A S}_{\mathcal{K}}$.

On the other hand, if we add ternary negative constraints, the cf 2 semantics will no longer coincide with the preferred semantics as shown in Example 10 
Example 10. Let $\mathcal{K}=(\mathcal{F}, \mathcal{R}, \mathcal{N})$ be a knowledge base such that $\mathcal{F}=\{a(m), b(m), c(m), d(m), e(m)\}$, $\mathcal{R}=\emptyset$ and $\mathcal{N}=\{\forall x(a(x) \wedge b(x) \wedge c(x) \rightarrow \perp), \forall x(e(x) \wedge d(x) \rightarrow \perp)\}$. The corresponding argumentation framework is composed of 161 attacks and the 20 following arguments:

- a0_0 : $(\{a(m)\},\{a(m)\})$

- $a 1_{-} 0:(\{b(m)\},\{b(m)\})$

- a2_2:( $\{a(m), b(m)\},\{a(m), b(m)\})$

- a3_0: $(\{c(m)\},\{c(m)\})$

- $a 4 \_2:(\{a(m), c(m)\},\{a(m), c(m)\})$

- $a 5 \_2:(\{b(m), c(m)\},\{b(m), c(m)\})$

- a6_0 : $(\{d(m)\},\{d(m)\})$

- $a 7 \_2:(\{a(m), d(m)\},\{a(m), d(m)\})$

- $\left.\left.\left.a 8 \_2:(\{b(m), d(m)\},\{b(m), d(m)\})\right), d(m)\right\}\right)$

- a9_6: $(\{a(m), b(m), d(m)\},\{a(m), b(m), d(m)\})$

- a10_2: $(\{c(m), d(m)\},\{c(m), d(m)\})$

- a11_6: $(\{a(m), c(m), d(m)\},\{a(m), c(m), d(m)\})$

- a12_6: $(\{b(m), c(m), d(m)\},\{b(m), c(m), d(m)\})$

- a13_0 : $(\{e(m)\},\{e(m)\})$

- a14_2: $(\{a(m), e(m)\},\{a(m), e(m)\})$

- a15_2: $(\{b(m), e(m)\},\{b(m), e(m)\})$

- a16_6: $(\{a(m), b(m), e(m)\},\{a(m), b(m), e(m)\})$

- a17_2: $(\{c(m), e(m)\},\{c(m), e(m)\})$

- a18_6: $(\{a(m), c(m), e(m)\},\{a(m), c(m), e(m)\})$

- a19_6: $(\{b(m), c(m), e(m)\},\{b(m), c(m), e(m)\})$

The preferred extensions will be composed of the following sets:

- $\varepsilon_{1}=\left\{a 0 \_0, a 1 \_0, a 2 \_2, a 6 \_0, a 7 \_2, a 8 \_2, a 9 \_6\right\}$

- $\varepsilon_{2}=\left\{a 0 \_0, a 3 \_0, a 4 \_2, a 6 \_0, a 7 \_2, a 10 \_2, a 11 \_6\right\}$

- $\varepsilon_{3}=\left\{a 1 \_0, a 3 \_0, a 5 \_2, a 6 \_0, a 8 \_2, a 10 \_2, a 12 \_6\right\}$

$-\varepsilon_{4}=\left\{a 0 \_0, a 1 \_0, a 2 \_2, a 13 \_0, a 14 \_2, a 15 \_2, a 16 \_6\right\}$

$-\varepsilon_{5}=\left\{a 0 \_0, a 3 \_0, a 4 \_2, a 13 \_0, a 14 \_2, a 17 \_2, a 18 \_6\right\}$

- $\varepsilon_{6}=\left\{a 1 \_0, a 3 \_0, a 5 \_2, a 13 \_0, a 15 \_2, a 17 \_2, a 19 \_6\right\}$

The set of cf2 extensions is the set $\left\{\varepsilon_{1}, \varepsilon_{2}, \varepsilon_{3}, \varepsilon_{4}, \varepsilon_{5}, \varepsilon_{6}, \varepsilon_{7}, \varepsilon_{8}\right\}$ with:

$-\varepsilon_{7}=\left\{a 0 \_0, a 1 \_0, a 3 \_0, a 6 \_0, a 7 \_2, a 8 \_2, a 10 \_2\right\}$

$-\varepsilon_{8}=\left\{a 0 \_0, a 1 \_0, a 3 \_0, a 13 \_0, a 14 \_2, a 15 \_2, a 17 \_2\right\}$

\subsection{Results for general knowledge bases}

In this subsection we consider the general case of knowledge bases composed of a set of facts, a set of rules and a set of negative constraints. Unfortunately, as the set of rules can completely change the argumentation framework, general results are much harder to obtain. It is easy to show that the link between the conflict graph (the hyper-graph generated by the negative constraints on the facts, potentially enriched with rules) bares 
no obvious link to the argumentation graph generated by the corresponding knowledge base. In the Appendix we show that there can be several argumentation frameworks associated with the same minimal conflict graph.

Despite the generality of the problem, we however present three graph theoretical structural results of argumentation graphs:

- First we show general structural properties of the graph: no self-attacking arguments, every argument is defended, having at least one cycle, etc.

- Second we demonstrate the presence of a complete directed sub-graph.

- Third, we show that preferred extensions are included into cf2 extensions but not the other way. Contrary to expectations, we show that the cf 2 semantics (originally designed to better handle cycles in graphs) is producing a set of arguments with an inconsistent base.

Let us start by making a few observations on the structure of the argumentation graph. Indeed, it is clear that not any graph can be obtained when constructing arguments from an existential rule knowledge base. First, we remark that an AF generated from a knowledge base $\mathcal{K}$ is always finite. Second, given the definition of an argument, we can also note that there are no self-attacking arguments in our framework:

Proposition 8. Let $\mathcal{A S}=(\mathcal{A}, \mathcal{C})$ be an argumentation framework s.t. there is an argu-

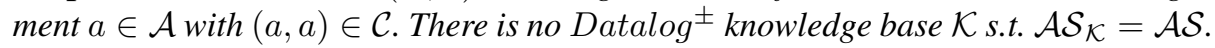

For every argument in the instantiated argumentation framework, there is a stable (resp. preferred and semi-stable) extension that contains it. Please note that the fact that there are no rejected arguments does not mean that the framework is not expressive as ranking-based semantics may be used to attach more fine-graded acceptability degrees to arguments.

Proposition 9. Let $\mathcal{A} \mathcal{S}_{\mathcal{K}}=(\mathcal{A}, \mathcal{C})$ be the corresponding $A F$ of $\mathcal{K}$. Then, for every argument $a \in \mathcal{A}$, there exists a preferred extension $\varepsilon \in \operatorname{Ext}_{p}\left(\mathcal{A} \mathcal{S}_{\mathcal{K}}\right)$ (resp. semi-stable extension $\varepsilon \in \operatorname{Ext}_{s s}\left(\mathcal{A S} \mathcal{S}_{\mathcal{K}}\right)$ and stable extension $\left.\varepsilon \in \operatorname{Ext}_{s}\left(\mathcal{A} \mathcal{S}_{\mathcal{K}}\right)\right)$ s.t. $a \in \varepsilon$.

We now focus on basic observations regarding attacks. First, no knowledge base can generate a framework where an argument $a$ is attacked by an unattacked argument $b$ :

Proposition 10. Let $\mathcal{A S}=(\mathcal{A}, \mathcal{C})$ be an argumentation framework. If there are two arguments $a, b$ s.t. $(b, a) \in \mathcal{C}$ and there does not exist $c \in \mathcal{A}$ s.t. $(c, b) \in \mathcal{C}$ then there is no Datalog ${ }^{ \pm}$knowledge base $\mathcal{K}$ s.t. $\mathcal{A S}_{\mathcal{K}}=\mathcal{A S}$.

In the next proposition, we prove the existence of particular arguments associated with a minimal conflict.

Proposition 11. Let $\mathcal{K}$ be a Datalog ${ }^{ \pm}$knowledge base and $\mathcal{A S}_{\mathcal{K}}=(\mathcal{A}, \mathcal{C})$ the corresponding instantiated $A F$ with $C$ a minimal conflict of $\mathcal{K}$ of size at least 2 . If $E, E^{\prime} \subset C$ such that $|E|=\left|E^{\prime}\right|=|C-1|$ and $E \neq E^{\prime}$ then the arguments $(E, E)$ and $\left(E^{\prime}, E^{\prime}\right)$ are in $\mathcal{A}$. 
Proof. By definition, we have that $E$ and $E^{\prime}$ are $\mathcal{R}$-consistent. Suppose that the argument $(E, E) \notin \mathcal{A}$, it means that there is $H \subset E$ and $E \subseteq \mathcal{C} \ell_{\mathcal{R}}^{*}(H)$ (minimality). It means that $(C \backslash E) \cup H$ is $\mathcal{R}$-inconsistent and $((C \backslash E) \cup H) \subset C$, contradiction.

If there is at least one minimal conflict $C$ of size at least 2 , then there is a cycle ${ }^{2}$ in the graph of the instantiated AF:

Proposition 12. If $\mathcal{K}$ is a Datalog ${ }^{ \pm}$knowledge base and $\mathcal{A} \mathcal{S}_{\mathcal{K}}=(\mathcal{A}, \mathcal{C})$ the corresponding instantiated $A F$ with $C$ a minimal conflict of $\mathcal{K}$ of size at least 2 then $\mathcal{A S}_{\mathcal{K}}$ has a cycle.

Proof. Since there is a minimal conflict of at least size 2 then we know from Proposition 11 that there are two arguments $(E, E)$ and $\left(E^{\prime}, E^{\prime}\right)$ in $\mathcal{A}$ such that $E, E^{\prime} \subset C, E \neq E^{\prime}$ and $|E|=\left|E^{\prime}\right|=|C-1|$. We have that $(E, E)$ attacks $\left(E^{\prime}, E^{\prime}\right)$ and conversely.

Minimal conflicts create a particular structure in the graph of the AF. For every minimal conflict of size $n$, there is a complete directed subgraph on $n$ nodes (i.e. a subgraph containing $n$ arguments where every argument attacks every argument except itself).

Proposition 13. Let $\mathcal{K}$ be a knowledge base and $\mathcal{A} \mathcal{S}_{\mathcal{K}}=(\mathcal{A}, \mathcal{C})$ the corresponding instantiated $A F$. For every minimal conflict $C$ of $\mathcal{K}$ s.t. $C \subseteq \mathcal{F}$, there exists a complete directed subgraph of $\mathcal{A} \mathcal{S}_{\mathcal{K}}$ with $|C|$ arguments.

Proof. We consider the case where $|C|>1$, otherwise it is obvious. Let us consider the set of arguments $\mathcal{A}_{C}=\{a \in \mathcal{A}|a=(S, S), S \subset C| S,|=| C \mid-1\}$. We know that $\left|A_{C}\right|=|C|$ and for all $a, b \in \mathcal{A}_{C}$ s.t. $a \neq b$, we have that $(a, b) \in \mathcal{C}$.

Let us now investigate the behaviour of the cf2 semantics on general Datalog ${ }^{ \pm}$ argumentation graphs. First, we show that the set of preferred extensions is included in the set of cf2 extensions. We know that in the general case, we have that a stable extension is also a cf2 extension [Gaggl and Woltran, 2013, Baroni et al., 2005].

Proposition 14. Let $\mathcal{A S}$ be a random $A F$, we have that $\operatorname{Ext}_{s t}(\mathcal{A S}) \subseteq \operatorname{Ext}_{c f 2}(\mathcal{A S})$.

Furthermore, since we are working in the setting of Datalog ${ }^{ \pm}$argumentation frameworks described in Croitoru and Vesic [2013], a basics result is that the set of preferred extension is equal to the set of stable semantics.

Proposition 15. Let $\mathcal{A S}$ be a Datalog ${ }^{ \pm} A F$, we have that $\operatorname{Ext}_{\text {st }}(\mathcal{A S})=\operatorname{Ext}_{p r}(\mathcal{A S})$.

We thus conclude that the set of preferred extensions is included the set of cf2 extensions for the case of Datalog ${ }^{ \pm}$AFs.

\footnotetext{
${ }^{2}$ We say that a tuple of arguments $\left(a_{1}, \ldots, a_{n}\right)$ is a cycle if and only if $a_{1} \mathcal{C} a_{2}, \ldots, a_{n-1} \mathcal{C} a_{n}$ and $a_{n} \mathcal{C} a_{1}$.
} 
Proposition 16. Let $\mathcal{A S}$ be a Datalog ${ }^{ \pm} A F$, we have that $\operatorname{Ext}_{p r}(\mathcal{A S}) \subseteq \operatorname{Ext}_{c f 2}(\mathcal{A S})$.

Note that this result is not true in general (for graphs not generated from Datalog ${ }^{ \pm}$ $\mathrm{KBs})$. Moreover, we highlight here that $\operatorname{Ext}_{c f 2}(\mathcal{A S}) \nsubseteq \operatorname{Ext}_{p r}(\mathcal{A S})$ in the Datalog ${ }^{ \pm}$ setting by providing the following counter-example.

Example 11. Let us consider the knowledge base $\mathcal{K}=(\mathcal{F}, \mathcal{R}, \mathcal{N})$ :

$$
\mathcal{F}=\{b(m), c(m), d(m), e(m)\}, \mathcal{R}=\{\forall x(f(x) \rightarrow b(x))\} \text { and } \mathcal{N}=\{\forall x(d(x) \wedge
$$
$b(x) \wedge c(x) \rightarrow \perp), \forall x(e(x), f(x) \rightarrow \perp)\}$.

The argumentation graph corresponding to this knowledge base is $\mathcal{A S}_{\mathcal{K}}=(\mathcal{A}, \mathcal{C})$ such that $\mathcal{A}$ is composed of:

- a0_0 : $(\{d(m)\},\{d(m)\})$

- $a 1 \_0:(\{b(m)\},\{b(m)\})$

- $a 1_{-} 1:(\{b(m)\},\{f(m)\})$

- $a 1 \_2:(\{b(m)\},\{b(m), f(m)\})$

- a2_2:(\{d(m),b(m)\},\{d(m),b(m)\})

- $a 2 \_4:(\{d(m), b(m)\},\{d(m), f(m)\})$

- a2_6 : $(\{d(m), b(m)\},\{d(m), b(m), f(m)\})$

- a3_0: $(\{c(m)\},\{c(m)\})$

- $a 4 \_2:(\{d(m), c(m)\},\{d(m), c(m)\})$

- $a 5 \_2:(\{b(m), c(m)\},\{b(m), c(m)\})$

- a5_5: $(\{b(m), c(m)\},\{c(m), f(m)\})$

- a5_6: $(\{b(m), c(m)\},\{b(m), c(m), f(m)\})$

- a6_0: $(\{e(m)\},\{e(m)\})$

- a7_2: $(\{d(m), e(m)\},\{d(m), e(m)\})$

- $a 8 \_2:(\{c(m), e(m)\},\{c(m), e(m)\})$

- a9_6: $(\{d(m), c(m), e(m)\},\{d(m), c(m), e(m)\})$

We have 3 preferred extensions $\operatorname{Ext}_{p r}=\left\{\varepsilon_{1}, \varepsilon_{2}, \varepsilon_{3}\right\}$ and $4 \mathrm{cf} 2$ extensions $\operatorname{Ext}_{c f 2}=$ Ext $_{p r} \cup\left\{\varepsilon_{4}\right\}$ with:

$$
\begin{aligned}
& -\varepsilon_{1}=\left\{a 0 \_0, a 1 \_0, a 1 \_1, a 1 \_2, a 2 \_2, a 2 \_4, a 2 \_6\right\} \\
& -\varepsilon_{2}=\left\{a 0 \_0, a 3 \_0, a 4 \_2, a 6 \_0, a 7 \_2, a 8 \_2, a 9 \_6\right\} \\
& -\varepsilon_{3}=\left\{a 1 \_0, a 1 \_1, a 1 \_2, a 3 \_0, a 5 \_2, a 5 \_5, a 5 \_6\right\} \\
& -\varepsilon_{4}=\left\{a 0 \_0, a 1 \_0, a 1 \_1, a 1 \_2, a 2 \_4, a 3 \_0, a 5 \_5\right\}
\end{aligned}
$$

We showed with Example 11 that the set of cf2 extensions are not included in the set of preferred extensions and thus not equal. Furthermore, contrary to expectations, the cf2 semantics (originally designed to better handle cycles in graphs) is producing a set of arguments with an inconsistent base. Indeed, the set $\varepsilon_{4}$ contains the arguments $a_{0 \_0}$ and $a_{5-5}$ which together form an inconsistent base. 


\section{Discussion}

In this paper we investigated the formal structural properties of argumentation graphs generated from Datalog ${ }^{ \pm}$knowledge bases.

We showed that for the case of argumentation frameworks originated from knowledge bases without rules, the dummy arguments are the result of facts that are not in the scope of any negative constraints and that their numbers are exponential w.r.t. these facts. Then, we proved that these frameworks possess a particular subgraph called $k$ copy graph which have symmetries in the form of automorphisms. Moreover, these symmetries can be transferred to the full argumentation framework without loss of generality. Next, we characterised the strong connectivity of the argumentation framework by explaining their structure. Lastly, we showed that the cf2 semantics coincides with the preferred and naive semantics in the case of argumentation frameworks generated from knowledge bases without rules and containing only binary negative constraints.

We then dealt with the case of argumentation frameworks generated from general knowledge bases with rules. We first showed general structural properties of the graph such as the absence of self-attacking arguments, the fact that every argument is defended and the presence of at least one cycle. Second we proved the presence of a complete directed sub-graph associated to each minimal conflict of the knowledge base. Third, we showed that preferred extensions are included into cf 2 extensions in this particular instantiation. Last, contrary to expectations, we proved by providing a counter-example that the cf2 semantics (originally designed to better handle cycles in graphs) is producing a set of arguments with an inconsistent base.

The significance of our results lies in the fact that this is the first paper highlighting the graph theoretical structural analysis of real world argumentation graphs. We believe that our thorough analysis will enable modellers to understand why and how the changes in the knowledge base can impact the structure of the argumentation framework. What's more, we feel that this paper could be useful for designing faster and better suited solvers for realistic argumentation graphs relying on their inherent structure.

Let us also make a note about the logical language used for instantiating the knowledge bases. Existential rules have been recently intensively investigated for their generalisation with respect to Description Logic fragments. Please note that certain structural results have also been shown to hold in the work of Arioua et al. [2017b]. However, their definition of argument is different from the one used in this paper (as our definition prevents unnecessary repeated arguments). We also note that using argumentation over existential rules has been shown to be of practical interest over existing approaches ([Hecham et al. , 2017a] $)$. Argumentation for handling inconsistency tolerant semantics enhance the human interaction ([Arioua and Croitoru, 2016]), can be used for practical applications in food science (|Arioua et al. |2016, 2017a]) or allow for alternative computation methods ([Yun and Croitoru. 2016]). Such techniques have been shown to have further implications with respect to human reasoning and bias detection ([Bisquert et al. 2016]). While the OBDA inspired restriction of inconsistency only coming from the facts could be too strong for certain applications, recently, argumentation inspired approaches that consider defeasible reasoning have been proposed ([Hecham et al. 2017b]). 
Future work will investigate the case of symmetries and strongly connectivity for argumentation graphs from general knowledge bases. Our goal is to obtain a complete characterisation of the argumentation graphs generated from a Datalog ${ }^{ \pm}$knowledge base.

\section{Appendices}

We first give the definition of a minimal conflict graph and show that there can be several argumentation frameworks associated with the same minimal conflict graph. This observation is highlighted with an example. We then characterise the arguments and attacks shared by every argumentation frameworks associated with the same minimal conflict graph.

Definition 8. The minimal conflict graph of an inconsistent knowledge $\mathcal{K}=(\mathcal{F}, \mathcal{R}, \mathcal{N})$ is a tuple $\left(\mathcal{F}, \mathcal{J}^{\prime}\right)$, where $\mathcal{J}^{\prime}=$ conflicts $(\mathcal{K})$. It can be represented with by an hypergraph where elements of $\mathcal{F}$ and elements of $\mathcal{J}^{\prime}$ represent nodes and hyper-edges respectively.

Please note that it is possible that two distinct argumentation frameworks have the same minimal conflict graph.

Example 12. Let $\mathcal{K}=(\mathcal{F}, \mathcal{R}, \mathcal{N})$ and $\mathcal{K}^{\prime}=\left(\mathcal{F}, \mathcal{R}^{\prime}, \mathcal{N}\right)$ be two knowledge bases with $\mathcal{F}=\{a(m), b(m)\}, \mathcal{R}=\emptyset, \mathcal{R}^{\prime}=\{\forall x(b(x) \rightarrow c(x)\}$ and $\mathcal{N}=\{\forall x(a(x) \wedge b(x) \rightarrow$ $\perp)\}$.

The argumentation framework $\mathcal{A} \mathcal{S}_{\mathcal{K}}=(\mathcal{A}, \mathcal{C})$ is composed of two arguments:

- $a_{1}:(\{a(m)\}, a(m))$

- $a_{2}:(\{b(m)\}, b(m))$

There are two attacks $\left(a_{1}, a_{2}\right)$ and $\left(a_{2}, a_{1}\right)$. However, the argumentation framework $\mathcal{A S}_{\mathcal{K}^{\prime}}=\left(\mathcal{A} \cup\left\{a_{3}, a_{4}\right\}, \mathcal{C}^{\prime}\right)$ is composed of two more arguments:

- $a_{3}:(\{b(m)\}, c(m))$

- $a_{4}:(\{b(m)\}, c(m) \wedge b(m))$

We have that $\mathcal{C}^{\prime}=\mathcal{C} \cup\left\{\left(a_{1}, a_{3}\right),\left(a_{1}, a_{4}\right),\left(a_{4}, a_{1}\right)\right\}$. We remind the reader that these two KBs have the same conflict graph.

Since different argumentation frameworks can have the same conflict graph, it gives us the intuition that there are similarities shared by all these argumentation frameworks.

Definition 9. The set of consistent subsets of a knowledge base $\mathcal{K}$ is defined as consistent $(\mathcal{K})=$ $\{X \subseteq \mathcal{F} \mid \nexists E \in \operatorname{conflicts}(\mathcal{K})$ and $E \subseteq X\}$.

Proposition 17. For every $X, X^{\prime} \in \operatorname{consistent}(\mathcal{K})$ such that there exists $C \in \operatorname{conflicts}(\mathcal{K})$ with $C \subseteq X \cup X^{\prime}$, we have that $\left(a_{1}, a_{2}\right) \in \mathcal{C}$ and $\left(a_{2}, a_{1}\right) \in \mathcal{C}$, where:

- $a_{1}:(X, X)$

- $a_{2}:\left(X^{\prime}, X^{\prime}\right)$

These arguments and attacks are shared by all the argumentation frameworks sharing the same minimal conflict graph. 


\section{Bibliography}

Leila Amgoud. Postulates for logic-based argumentation systems. Int. J. Approx. Reasoning, 55(9):2028-2048, 2014.

Abdallah Arioua and Madalina Croitoru. A dialectical proof theory for universal acceptance in coherent logic-based argumentation frameworks. In ECAI 2016 - 22nd European Conference on Artificial Intelligence, 29 August-2 September 2016, The Hague, The Netherlands - Including Prestigious Applications of Artificial Intelligence (PAIS 2016), pages 55-63, 2016.

Abdallah Arioua, Madalina Croitoru, and Patrice Buche. DALEK: A tool for dialectical explanations in inconsistent knowledge bases. In Computational Models of Argument - Proceedings of COMMA 2016, Potsdam, Germany, 12-16 September, 2016., pages 461-462, 2016.

Abdallah Arioua, Patrice Buche, and Madalina Croitoru. Explanatory dialogues with argumentative faculties over inconsistent knowledge bases. Expert Syst. Appl., 80:244262, 2017.

Abdallah Arioua, Madalina Croitoru, and Srdjan Vesic. Logic-based argumentation with existential rules. Int. J. Approx. Reasoning, 90:76-106, 2017.

Jean-François Baget, Michel Leclère, Marie-Laure Mugnier, and Eric Salvat. On rules with existential variables: Walking the decidability line. Artif. Intell., 175(910):1620-1654, 2011.

Pietro Baroni, Massimiliano Giacomin, and Giovanni Guida. SCC-recursiveness: a general schema for argumentation semantics. Artif. Intell., 168(1-2):162-210, 2005.

Pietro Baroni, Martin Caminada, and Massimiliano Giacomin. An introduction to argumentation semantics. Knowledge Eng. Review, 26(4):365-410, 2011.

Meghyn Bienvenu. On the Complexity of Consistent Query Answering in the Presence of Simple Ontologies. In Proceedings of the Twenty-Sixth AAAI Conference on Artificial Intelligence, July 22-26, 2012, Toronto, Ontario, Canada., 2012.

Pierre Bisquert, Madalina Croitoru, Florence Dupin de Saint-Cyr, and Abdelraouf Hecham. Substantive irrationality in cognitive systems. In ECAI 2016 - 22nd European Conference on Artificial Intelligence, 29 August-2 September 2016, The Hague, The Netherlands - Including Prestigious Applications of Artificial Intelligence (PAIS 2016), pages 1642-1643, 2016.

Andrea Calì, Georg Gottlob, and Thomas Lukasiewicz. A general datalog-based framework for tractable query answering over ontologies. In Proceedings of the TwentyEigth ACM SIGMOD-SIGACT-SIGART Symposium on Principles of Database Systems, PODS 2009, June 19 - July 1, 2009, Providence, Rhode Island, USA, pages 77-86, 2009.

Martin Caminada and Leila Amgoud. On the evaluation of argumentation formalisms. Artif. Intell., 171(5-6):286-310, 2007.

Federico Cerutti, Paul E. Dunne, Massimiliano Giacomin, and Mauro Vallati. Computing Preferred Extensions in Abstract Argumentation: A SAT-Based Approach. In Theory and Applications of Formal Argumentation - Second International Workshop, 
TAFA 2013, Beijing, China, August 3-5, 2013, Revised Selected papers, pages 176193, 2013.

Madalina Croitoru and Srdjan Vesic. What Can Argumentation Do for Inconsistent Ontology Query Answering? In Scalable Uncertainty Management - 7th International Conference, SUM 2013, Washington, DC, USA, September 16-18, 2013. Proceedings, pages 15-29, 2013.

Phan Minh Dung. On the Acceptability of Arguments and its Fundamental Role in Nonmonotonic Reasoning, Logic Programming and n-Person Games. Artif. Intell., 77(2):321-358, 1995.

Sarah Alice Gaggl and Stefan Woltran. The cf2 argumentation semantics revisited. J. Log. Comput., 23(5):925-949, 2013.

Abdelraouf Hecham, Abdallah Arioua, Gem Stapleton, and Madalina Croitoru. An empirical evaluation of argumentation in explaining inconsistency tolerant query answering. In Description Logics, 30th International Workshop, DL 2017, Montpellier, France, 2017.

Abdelraouf Hecham, Madalina Croitoru, and Pierre Bisquert. Argumentation-based defeasible reasoning for existential rules. In Proceedings of the 16th Conference on Autonomous Agents and MultiAgent Systems, AAMAS 2017, São Paulo, Brazil, May 8-12, 2017, pages 1568-1569, 2017.

Jean-Marie Lagniez, Emmanuel Lonca, and Jean-Guy Mailly. Coquiaas: A constraintbased quick abstract argumentation solver. In Tools with Artificial Intelligence (ICTAI), 2015 IEEE 27th International Conference on, pages 928-935. IEEE, 2015.

Domenico Lembo, Maurizio Lenzerini, Riccardo Rosati, Marco Ruzzi, and Domenico Fabio Savo. Inconsistency-Tolerant Semantics for Description Logics. In Web Reasoning and Rule Systems - Fourth International Conference, RR 2010, Bressanone/Brixen, Italy, September 22-24, 2010. Proceedings, pages 103-117, 2010.

Matthias Thimm, Serena Villata, Federico Cerutti, Nir Oren, Hannes Strass, and Mauro Vallati. Summary Report of The First International Competition on Computational Models of Argumentation. AI Magazine, 37(1):102, 2016.

Michaël Thomazo and Sebastian Rudolph. Mixing Materialization and Query Rewriting for Existential Rules. In ECAI 2014 - 21st European Conference on Artificial Intelligence, 18-22 August 2014, Prague, Czech Republic - Including Prestigious Applications of Intelligent Systems (PAIS 2014), pages 897-902, 2014.

Bruno Yun and Madalina Croitoru. An argumentation workflow for reasoning in ontology based data access. In Computational Models of Argument - Proceedings of COMMA 2016, Potsdam, Germany, 12-16 September, 2016., pages 61-68, 2016.

Bruno Yun, Madalina Croitoru, and Pierre Bisquert. Are ranking semantics sensitive to the notion of core? In Autonomous Agents and Multiagent Systems - Proceedings of AAMAS 2017, Sao Paulo, Bresil, 8-12 May, 2017, 2017.

Bruno Yun, Srdjan Vesic, Madalina Croitoru, Pierre Bisquert, and Rallou Thomopoulos. A structural benchmark for logical argumentation frameworks. In Proceedings of the 20th International Symposium on Intelligent Data Analysis (2017), 2017.

Bruno Yun, Madalina Croitoru, Srdjan Vesic, and Pierre Bisquert. A structural benchmark for logical argumentation frameworks. In Proceedings of the 17th Conference on Autonomous Agents and MultiAgent Systems, AAMAS, 2018. 
Heng Zhang, Yan Zhang, and Jia-Huai You. Expressive Completeness of Existential Rule Languages for Ontology-Based Query Answering. In Proceedings of the Twenty-Fifth International Joint Conference on Artificial Intelligence, IJCAI 2016, New York, NY, USA, 9-15 July 2016, pages 1330-1337, 2016. 